\title{
Effets des gravières sur le comportement hydrodynamique des nappes d'eau souterraines
}

PAR

\author{
P. Peaudecerf \\ Ingénieur au B.R.G.M.
}

Les énormes besoins en matériaux de construction et de remblai ont transformé bien des plaines alluviales de notre pays en une succession d'excavations. Dans la plupart des cas, ces formations alluviales constituent des aquifères de bonnes caractéristiques et les excavations atteignent le niveau de la nappe. Elles constituent alors autant de plans d'eau.

En règle générale, dans les années passées, on a tenté de maintenir ces extractions de graviers hors des zones où les eaux souterraines étaient exploitées. Les ressources en matériau s'amenuisant au cours des années, la pression des besoins fait que les exploitants des sablières et des gravières tentent de s'implanter bien plus près des captages d'eau. Ce voisinage, cette exploitation simultanée de l'eau et du gravier sont-ils compatibles? Avant d'avancer une réponse, il convient de bien mesurer les effets positifs ou négatifs de la présence des gravières au sein des nappes. Elles peuvent influer sur le comportement hydrodynamique des eaux souterraines, sur les niveaux et les écoulements. Suivant leurs positions par rapport au système aquifère, elles tendent à augmenter l'alimentation ou le drainage des nappes. Par leurs éventuelles communications avec les cours d'eau, par le colmatage de leurs berges, elles peuvent constituer un cheminement privilégié ou, au contraire, un obstacle à l'écoulement des eaux souterraines.

Mais ce sont les effets des gravières sur la qualité des eaux qui viennent le plus naturellement à l'esprit. En contact avec l'atmosphère et sous l'influence du rayonnement solaire, les eaux des gravières se peuplent d'organismes animaux ou végétaux. Leurs caractères chimiques ou biologiques peuvent être notablement modifiés. Ces phénomènes ont fait l'objet d'études pleines d'intérêt.

D'autre part, véritable mise à nu de la nappe, les carrières constituent au sein des aquifères une zone très vulnérable à la pollution. Même dans les cas où le dépôt des matières polluantes, comme les déchets industriels ou ménagers, est officiellement interdit, il est en pratique très difficile de l'empêcher totalement. La présence de gravières en eau multiplie les risques de pollution des eaux souterraines.

Or, si l'on fait appel en priorité aux eaux souterraines pour l'alimentation des populations urbaines ou rurales, c'est principalement à cause de leurs qualités biologiques et chimiques.

Mais ces pollutions tant redoutées ne sont à craindre que si des transferts notables peuvent avoir lieu entre le plan d'eau libre et la nappe.

Ainsi, que ce soit pour évaluer les perturbations des niveaux et des écoulements de la nappe consécutives au creusement de gravières, ou encore pour prévoir la vulnérabilité des réserves en eau souterraine à une éventuelle pollution, il est nécessaire de bien connaître l'hydrodynamisme des systèmes gravière-nappe.

Dans les lignes qui suivent, nous nous proposons de passer rapidement en revue les facteurs les plus importants qu'il faudra prendre en compte pour cette étude. A cette occasion, nous évoquerons leurs effets principaux. Ensuite, nous étudierons les moyens dont dispose l'hydrogéologue pour évaluer ces paramètres et prévoir leurs effets. Nous illustrerons notre propos par des références à des études récentes ou actuelles. 


\section{Les paramètres principaux du système nappe-gravière}

Les effets du creusement d'une gravière sur les niveaux de la nappe, les perturbations apportées à son écoulement ou encore les débits d'échange entre gravière et nappe dépendent des facteurs géométriques ou physiques suivants :

\section{IMPORTANCE DU COLMATAGE DES BORDS ET DU FOND}

Ce premier paramètre évoqué constitue un facteur essentiel de l'influence d'une gravière sur une nappe. Habituellement, seuls le fond et les berges avals connaissent un colmatage durable. Ses effets sont évidents comme nous l'avons schématisé à la figure 1 :

- une carrière non colmatée accroît les écoulements souterrains: la partie de l'aquifère à son amont est rabattue, à l'aval les niveaux sont relevés. Il se produit une convergence des filets fluides vers l'excavation. Les débits d'échange vers la nappe sont maximaux;

- au contraire, si les berges sont colmatées, la carrière constitue pour les écoulements souterrains un obstacle qui doit être contourné. Les niveaux de la nappe s'élèvent à l'amont, ils sont rabattus à l'aval. Il peut même y avoir un débordement du plan d'eau de la gravière. Les débits d'échange vers la nappe et les risques de pollution sont donc minimes.

Le colmatage est le résultat de phénomènes divers et mal connus. Il peut être provoqué par le dépôt des matériaux les plus fins lors de l'extraction des sables et graviers, par un processus physico-chimique ou même biologique. Dans l'état actuel des connaissances, et en attendant des études poussées de ces phénomènes, il est très difficile de prévoir son intensité et ses variations au cours des années à venir. En cas de besoin, on peut tenter d'accélérer ce colmatage par des procédés mécaniques.

\section{PÉNÉTRATION DE L'EXCAVATION}

On ne s'intéresse qu'aux gravières atteignant le niveau de la nappe. On peut considérer les deux cas de la figure 2. $\mathrm{Si}$ les berges et le fond ne sont pas colmatés, la profondeur de pénétration n'aura pratiquement pas d'effet car les écoulements dans les formations restées en place en dessous seront négligeables par rapport à ceux passant dans la carrière. Au contraire, pour les carrières colmatées, les écoulements ne seront possibles que dans la tranche des matériaux restés en place et la transmissivité effective sera diminuée en proportion de la pénétration de l'excavation.

\section{EMPLACEMENT DES GRAVIERES PAR RAPPORT AU SYSTẼME AQUIFÈRE}

La position de ce plan d'eau libre importe bien évidemment. En amont de la zone aquifère considérée, il améliore son alimentation ou la diminue suivant le degré du colmatage des bords. A cet emplacement les risques de pollution sont les plus importants. Au contraire, à l'aval de la nappe, c'est son drainage qui est augmenté ou diminué suivant ce colmatage.

\section{RELATIONS AVEC LES COURS D'EAU}

Les relations des plans d'eau des excavations avec les cours d'eau permettent de maintenir, au milieu du système aquifère, un niveau imposé avec tout ce que cela entraîne comme conséquences sur les écoulements et, notamment, l'augmentation du drainage ou de l'alimentation de la nappe.

La position même de ces relations peut avoir un effet important. Considérons, par exemple, le cas schématisé à la figure 3 , d'une sablière allongée parallèlement à un cours d'eau de pente non négligeable. On suppose qu'il existe une nappe dont l'écoulement général est approximativement parallèle à celui du cours d'eau. Suivant que le canal de liaison sera situé à l'amont ou à l'aval, les débits d'échange se feront de la gravière vers la nappe ou le contraire et, en conséquence, on observera une alimentation ou un drainage accru de la nappe.

\section{FORMES ET DIMENSIONS DES EXCAVATIONS; ORIENTATION PAR RAPPORT A L'ÉCOULEMENT DE LA NAPPE}

Une carrière en eau impose, au milieu de la nappe, un niveau pratiquement horizontal sur toute sa surface. On conçoit que son influence dépende de sa forme et de ses dimensions.

Une excavation de forme allongée (cf. fig. 4), dont la plus grande dimension est perpendiculaire à l'écoulement naturel des eaux souterraines, aura peu d'effet sur les écoulements. Au contraire, si elle est creusée parallèlement à ces écoulements, les rabattements provoqués à l'amont et les élévations du niveau à l'aval (ou les risques de débordements du plan d'eau en cas de berges colmatées) seront très importants.

Dans cette dernière configuration, et comme l'expliquent les figures 5 et 6 , le morcellement de l'exploitation peut permettre d'éviter ces débordements même si le colmatage des berges et la topographie ont tendance à les provoquer.

\section{CARACTÉRISTIQUES DU SYSTÈME AQUIFÈRE}

Le comportement de la nappe sous l'influence d'éventuelles gravières dépendra bien évidemment de ses caractéristiques propres.

Il nous suffira d'énumérer quelques-uns de ces paramètres omniprésents dans les études hydrogéologiques: la piézométrie, l'extension de la nappe, ses limites et leurs natures, les paramètres hydrodynamiques dont l'hétérogénéité peut jouer un rôle important. Il faut souligner que la propagation d'une pollution à partir d'une gravière peut être influencée par les éventuelles différences des perméabilités de chaque horizon. Ce phénomène est d'ailleurs commun à tous les transferts de masse dans les milieux poreux stratifiés.

Nous pouvons aussi noter qu'il faudra évidemment considérer la profondeur de l'eau et la topographie pour évaluer les risques de débordement.

Enfin, limplantation d'un plan d'eau est la cause de pertes par évaporation, mais celles par l'évapotranspiration, 
Plan

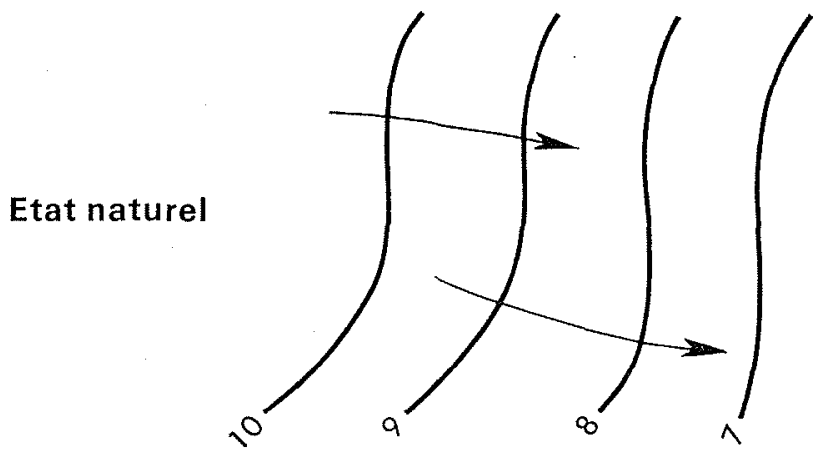

Piézométrie naturelle

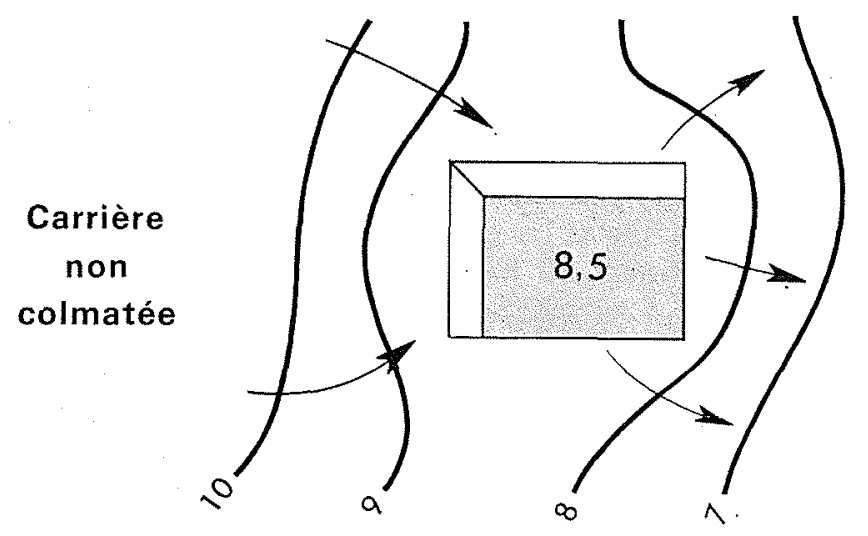

Convergence des écoulements

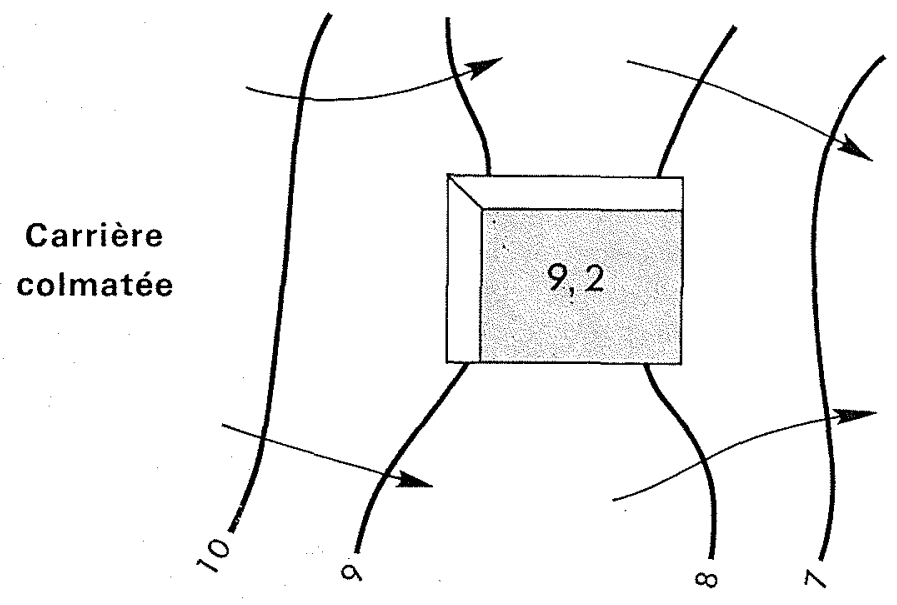

Obstacle

\section{Coupe}

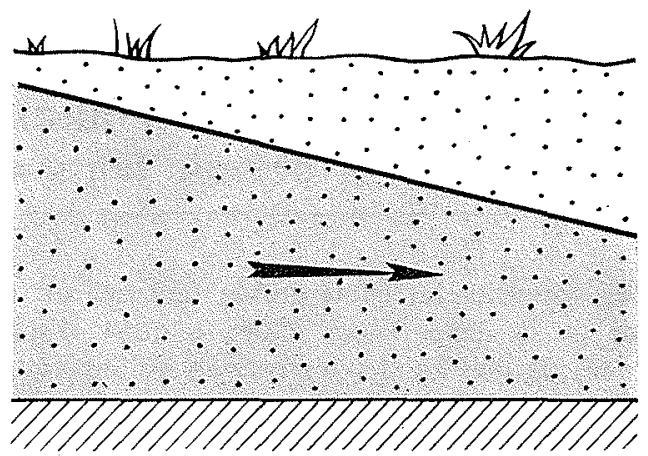

1/ Effets du colmatage de la gravière.

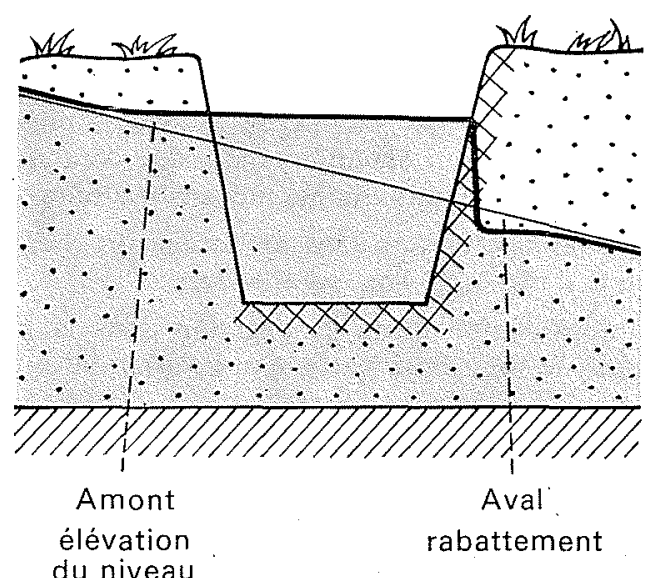

élévation du niveau 
due aux végétaux qui croissaient sur le sol disparu, peuvent être plus importantes. D'autre part, le décapage de couches superficielles imperméables peut favoriser l'alimentation par les pluies, et la création de bassin peut accroître l'infiltration d'eaux de ruissellement. Ces phénomènes dépendent de la surface des exploitations. Ils sont habituellement de faible importance.

\section{Moyens disponibles pour évaluer ces paramètres et prévoir leurs effets}

Pour apprécier les effets de ces facteurs sur le comportement de la nappe, nous avons à notre disposition des méthodes de mesure et d'interprétation. Sur certains points cependant, il apparaît nécessaire d'approfondir la méthodologie.

\section{MÉTHODES DE MESURE}

Il n'est pas utile d'évoquer les paramètres géométriques. Quant aux paramètres hydrogéologiques, leurs relevés et mesures sont tellement utilisés, qu'on peut ne pas les rappeler ici. Il faut cependant faire une mention spéciale pour la mesure des perméabilités : répartition verticale des perméabilités des différents horizons et surtout évaluation du colmatage.

- Le relevé systématique des perméabilités sur une même verticale est habituel en géotechnique. Les méthodes de mesure dans les forages, basées sur l'injection ou le pompage d'eau, sont relativement coûteuses. Ces coûts peuvent ne pas être acceptables dans les études des influences gravière-nappe. Dans les forages adéquats, un relevé des vitesses au micromoulinet pourra donner des résultats suffisants pour évaluer l'hétérogénéité verticale des perméabilités. Il s'agit sans doute de la méthode la plus appropriée.

- On a vu plus haut combien le colmatage des berges et du fond de la gravière était le paramètre essentiel. A défaut de bien connaître le processus de sa formation, il apparaît au moins nécessaire de savoir le mesurer, sur la gravière elle-même ou sur des gravières plus anciennes, pour connaitre son évolution dans des conditions comparables. Or, nous avons comme moyens pratiques de mesure :

- le pompage d'essai dans un forage proche du bord du plan d'eau que l'on veut tester;

- l'interprétation de la propagation dans la nappe d'une onde de pression à partir d'une variation du plan d'eau;

- le prélèvement d'échantillons et leur analyse en laboratoire.

Disons tout de suite que cette dernière technique est très délicate, car elle consiste à prélever des échantillons non consolidés en milieu aqueux. Elle présente l'inconvénient d'être très localisée. La seconde méthode est rarement utilisable car les fluctuations de la nappe ne dépendent pas directement de celles du plan d'eau libre voisin, mais le plus souvent elles ont les unes et les autres la même cause, les précipitations. Seuls les cas où la carrière est en relation avec un cours d'eau de quelque importance peuvent être traités ainsi. On obtient la diffusivité moyenne de la partie du milieu poreux située entre le forage de mesure et le plan d'eau libre.

Enfin, les pompages d'essai constituent la base de la méthode la plus générale et la plus sûre. Leur interprétation n'est pas toujours aisée. Un colmatage très poussé se détectera bien évidemment par l'apparition des effets d'une barrière étanche. Dans le cas contraire, on observera une stabilisation des niveaux. Le temps d'apparition de cette stabilisation nous permettra de calculer la «distance fictive » du plan d'eau si l'on suppose le milieu aquifère homogène. S'il n'y a pas de colmatage, cette distance est exactement égale à la distance séparant le plan d'eau du forage. L'intensité du colmatage sera donc matérialisée par cette «distance fictive ». Au cours des interprétations ultérieures sa valeur sera directement utilisable dans les calculs ou les modèles.

Il n'en demeure pas moins qu'un effort important doit être effectué pour connaître le processus de formation du colmatage et pour mettre au point des méthodes pratiques pour le mesurer.

\section{MÉTHODES D'INTERPRÉTATION}

Il s'agit d'évaluer le comportement du système gravièrenappe. Suivant la complexité du problème un simple calcul suffira ou il faudra faire appel à un modèle de simulation. La gamme importante des modèles en hydrogéologie permet de simuler toutes les conditions possibles.

On peut souligner les grands services que peuvent rendre dans ce but les modèles analogiques par la technique du papier conducteur. Leur simplicité d'emploi, leur coût très faible, la faculté de reproduire aisément les formes géomètriques des gravières et des limites, en font des outils très appropriés à ce problème. Localement l'homogénéité des transmissivités peut être souvent acceptée. Mais la simulation des colmatages manque évidemment de finesse. Ces paramètres restent pour la plupart des cas mal connus et la simulation des conditions extrêmes est parfois suffisante.

$\mathrm{Si}$ on recherche plus de précision, pour un aménagement plus important par exemple, on peut mettre en cuvre des techniques plus sophistiquées. On peut citer les modèles mathématiques à mailles régulières ou de dimensions variées pouvant tenir compte des effets éventuels de nappe libre. Ils peuvent simuler des débordements de la nappe et du plan d'eau. Les données géométriques et hydrogéologiques à rassembler sont évidemment plus nombreuses.

Les simulations peuvent être limitées au régime permanent. Elles peuvent être suivies de la simulation de la propagation d'un front de pollution à partir d'un point quelconque de la nappe.

Ces modèles permettent de répondre complètement aux questions telles que les suivantes:

- Quel sera le rabattement de la nappe à l'amont du plan d'eau? L'élévation du niveau à l'aval ?

- Quelle sera l'influence de la gravière en eau sur tel captage, sur son niveau ou son débit?

- Y a-t-il des risques de débordement du plan d'eau?

- Quelle sera la proportion du débit de captage provenant du plan d'eau ? Quel est le temps de cheminement de l'eau entre la gravière et le captage ?

- Etc. 


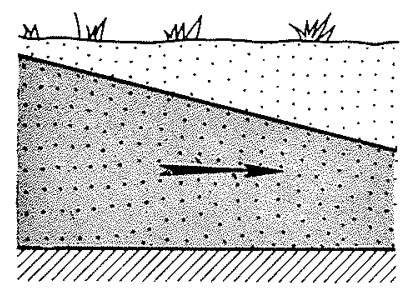

Transmissivité naturelle To

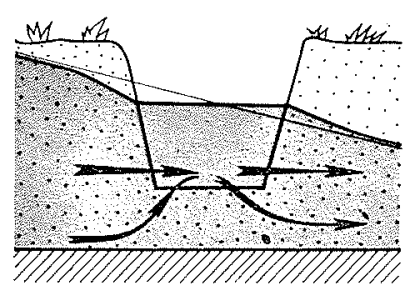

Carrière non colmatée $T=\infty$

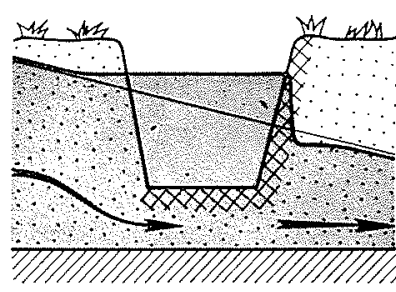

Carrière colmatée $T<T_{0}$

2/ Effet de la pénétration de l'excavation.

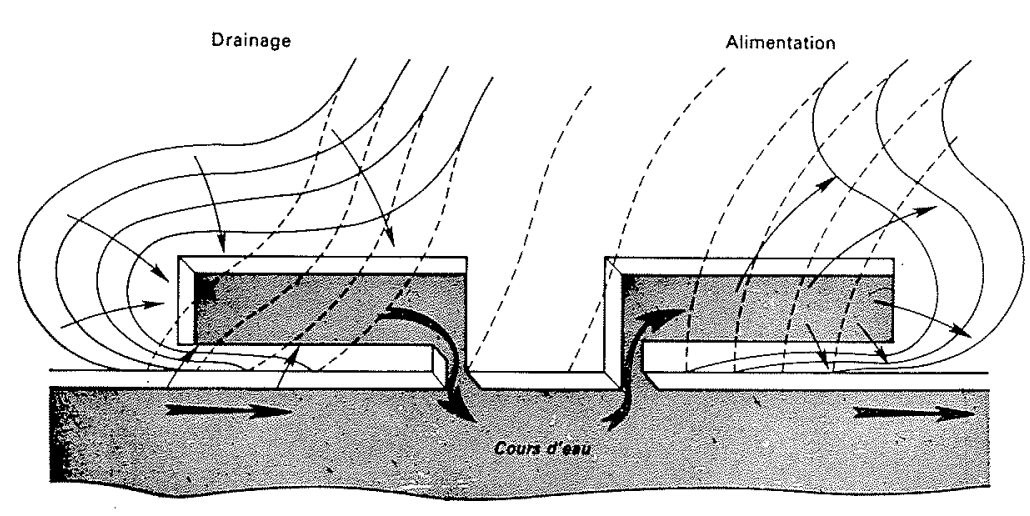

3/ Effets des relations avec les cours d'eau.

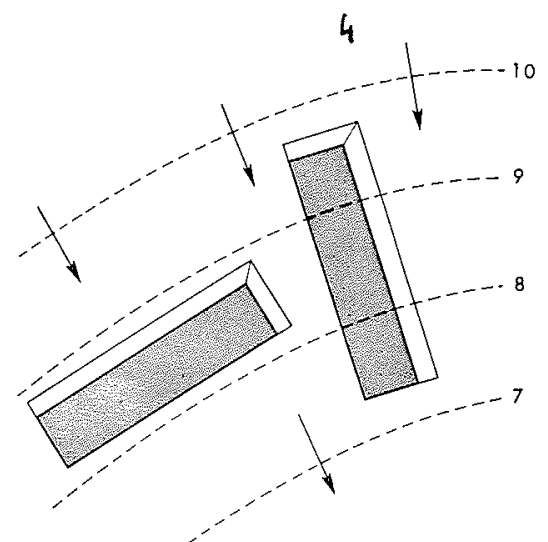

4/ Exemple d'orientation des gravières.

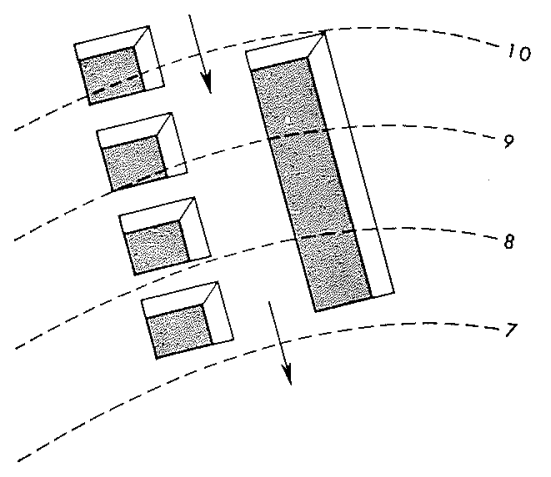

5/ Exemple de morcellement des excavations.

6/ Effets du morcellement des gravières colmatées.
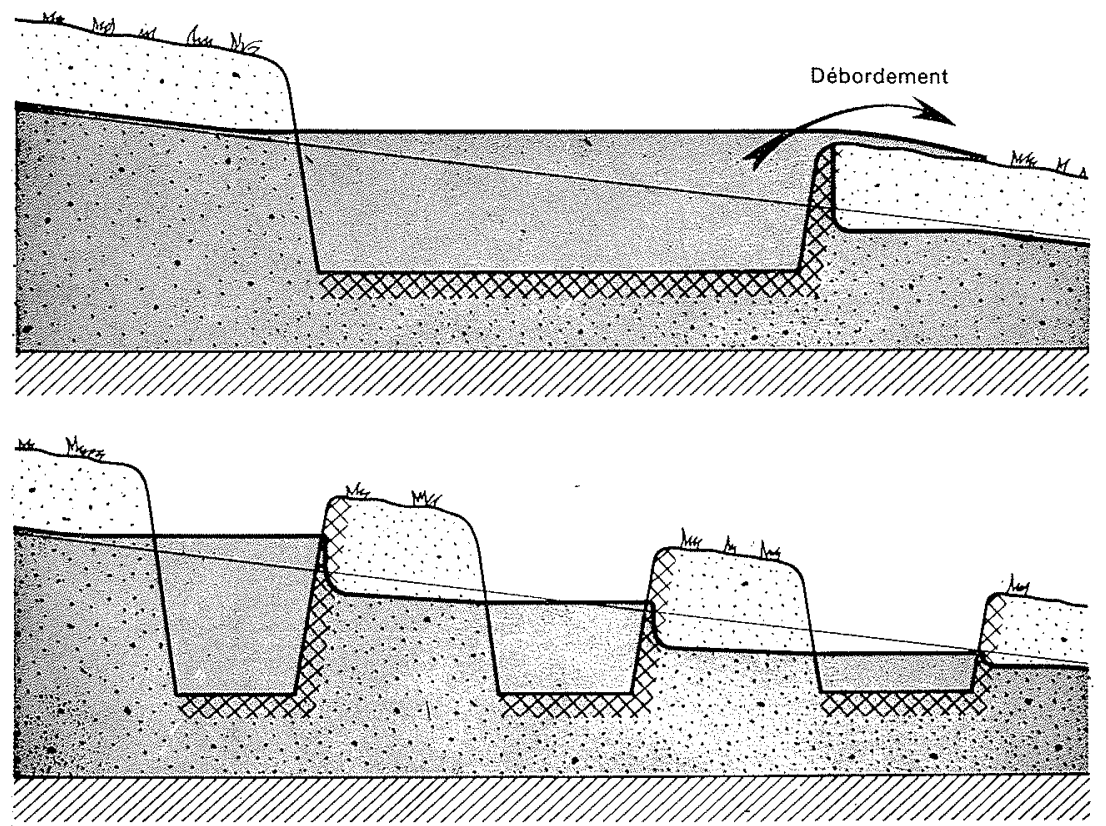

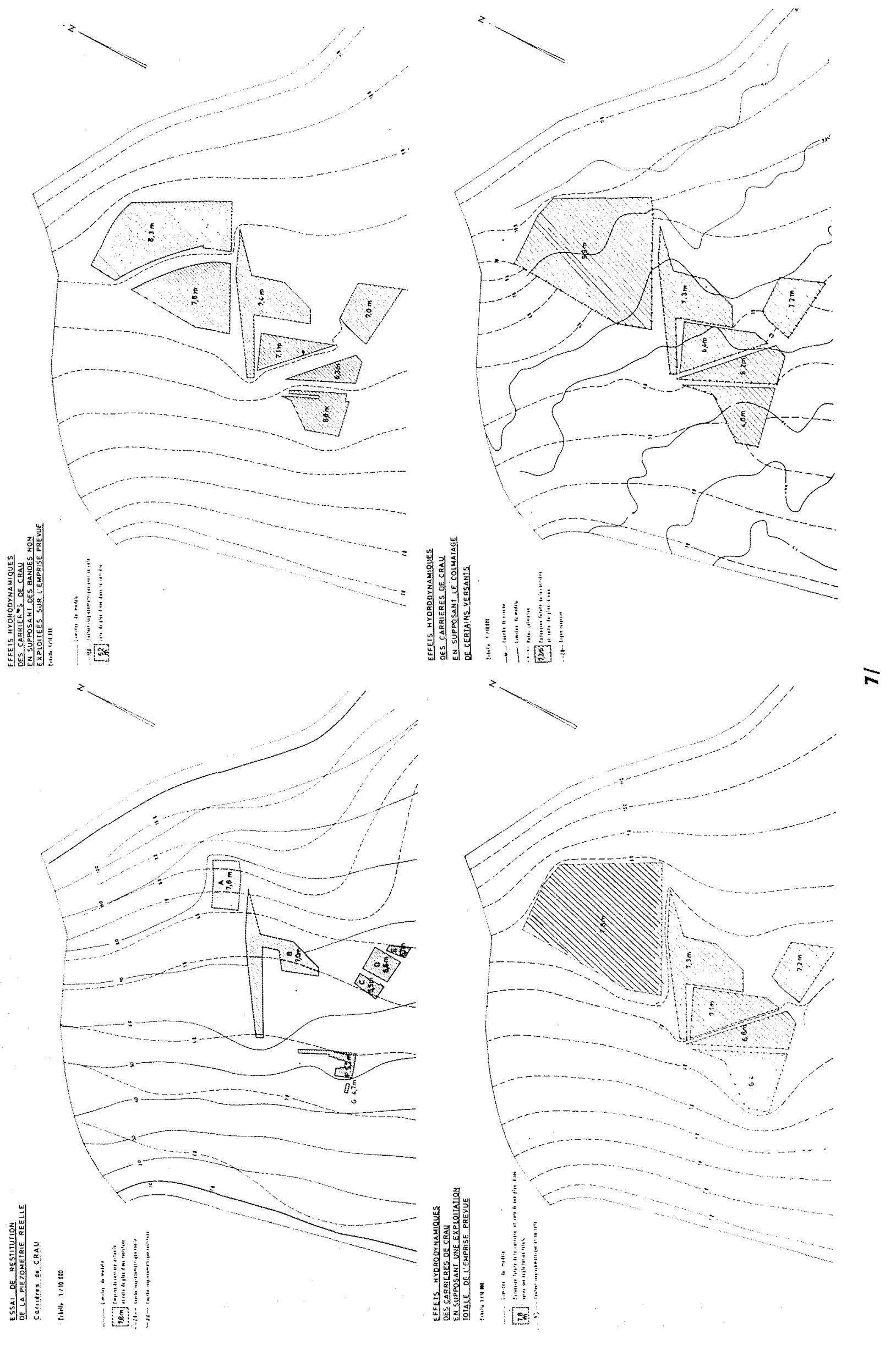


\section{Exemples d'études}

Pour illustrer ces propos, considérons les études très différentes suivantes:

\section{GRAVIËRES EN CRAU (*)}

Prenons, par exemple, le cas qui s'est posé lorsqu'il s'est agi d'étendre des gravières en Crau. Les alluvions de la Crau possèdent à la fois une nappe remarquable par ses dimensions et ses qualités et des réserves en graviers non moins remarquables. Ces ressources en eau ou en graviers ont d'autant plus de valeur qu'elles se trouvent à proximité de la zone industrielle de Fos en cours d'aménagement.

La zone qui nous intéresse est située à la pointe Sud de la Crau. Des gravières sont en cours d'exploitation. Elles atteignent très rarement le niveau de la nappe, mais il est prévu d'exploiter plus profondément la couche utile. Les autorités compétentes se sont donc posé les questions suivantes :

$1^{\circ}$ Quels seront les niveaux des plans d'eau du fait de la platitude de la topographie ? N'y aura-t-il pas de risque de débordement?

\section{$2^{\circ}$ Quels seront les effets sur la nappe?}

A la suite des nombreuses études qui y ont été consacrées, la nappe est assez bien connue, notamment sa piézométrie et l'ordre de grandeur de ses transmissivités. Afin de répondre à ces questions, on a construit un modèle analogique simple de la nappe par la technique du papier conducteur. Différentes configurations des plans d'eau ont été simulées:

- en laissant des bandes de terrain non exploitées;

- en supposant que les berges avals étaient ou non colmatées.

Les niveaux de la nappe et des plans d'eau ont été ainsi déterminés comme on peut le voir à la figure 7 .

Contrairement à ce qui se passe pour un plan d'eau unique, c'est lorsqu'il y a colmatage des berges avals que les risques de débordement sont les plus grands.

\section{PLAINE DE TOULOUSE (**)}

Cette étude, au contraire, a un but beaucoup plus général. Elle doit permettre de définir les modalités d'une exploitation rationnelle de l'eau et des matériaux sur $80 \mathrm{~km}$ de la vallée de la Garonne au voisinage de la ville de Toulouse.

(5) Etude entreprise à l'instigation du Service des Mines de Marseille.

(*) Etude réalisée à la demande de l'Agence Financière de Bassin Adour-Garonne, du Ministère de l'Agriculture, Direction Départementale de la Haute-Garonne et du Ministère du Développement Industriel et Scientifique, Arrondissement minéralogique de Toulouse.
Cette étude est en cours d'exécution, elle doit se dérouler en trois phases:

$1 /$ Etude systématique, sur des plans d'eau existants, des communications avec la nappe:

Près de la berge aval de quatre gravières d'âges différents, des stations d'essai ont été implantées: forages et piézomètres. Des pompages d'essai ont été effectués sur chacune d'elles dans le but de détecter et de mesurer d'éventuels colmatages. Il est prévu de tenter de corréler les caractéristiques des gravières et notamment leur âge avec le degré de colmatage.

Nous sommes conscients que les relations mises en évidence ne pourront être appliquées sans vérification à d'autres systèmes aquifères. Par contre, le test des méthodes présente un intérêt tout à fait général.

\section{2) Simulation d'une zone limitée:}

Dans le but de définir les modalités de mise en exploitation de gravières au voisinage d'un captage isolé, une étude complète d'une zone de la nappe est effectuée. Il s'agit d'une région encore peu exploitée mais contenant un captage d'eau potable. Après l'étude hydrogéologique complète de la zone, un modèle mathématique est élaboré. Il doit permettre:

- la détermination de la zone d'appel du captage;

- la définition des dimensions et des emplacements des gravières qui sont compatibles avec la présence du captage;

- l'évaluation des vitesses d'écoulement des eaux vers le captage.

3/ Application des phases précédentes à la définition de règles de répartition des captages et des gravières sur l'ensemble de la plaine et, en particulier:

- emplacements et dimensions des gravières;

- détermination des captages actuellement menacés;

- zones où l'exploitation des matériaux peut être faite sans entraves.

$$
\text { : }
$$

En conclusion, nous pouvons souligner le nombre et l'importance des paramètres qui président aux relations nappes-gravières, ce qui multiplie les cas d'espèces.

Il faut donc se garder de toute affirmation à prétention générale sur l'effet des gravières: elles ne sont en général ni bénéfiques - et à favoriser - ni préjudicielles - et à proscrire. Cela dépend des conditions locales aussi bien que de la configuration donnée aux gravières et de leur position.

Les questions que l'on se pose au sujet de ce voisinage ne peuvent être réglées sans des études cas par cas. Bien que sur certains points essentiels des études méthodologiques seraient très utiles, on dispose de méthodes de mesure et d'interprétation suffisantes pour prévoir, au moins d'une manière approchée, le comportement du système. 


\section{Discussion}

Président : M. THÉVENIN

M. le Président remercie $M$. Peaudecerf de son intéressante communication qui traite d'un sujet de grande actualité.

En effet, dit-i', il faut des quantités croissantes de matériaux de construction, et, en attendant d'autres solutions, il faut aller les chercher dans les plaines alluviales en faisant en sorte que l'exploitation de ces matériaux ne compromette pas l'exploitation des nappes nécessaires à l'alimentation humaine et ne nuisent pas à l'environnement. Afin de préserver les différents intérêts en cause on est amené maintenant, dans les grandes vallées, à prévoir un véritable «zoning» qui fait sa place à l'exploitation des graviers et du sable, mais de facon que les richesses en eau de ces vallées ne soient pas compromises.

M. le Président ouvre ensuite la discussion.

M. Clouet D'Orval (BURGEAP, Paris) pose la question ci-après :
A propos du colmatage, M. Peaudecerf a supposé implicitement, dans son exposé, que celui-ci n'était possible que dans le cas où l'écuulement va du plan d'eau vers la nappe. Est-ce toujours le cas ? Le colmatage ne peut-il se produire par simple dépôt gravitaire sur le fond ou les berges en pente de la gravière, indépendamment du sens de l'écoulement d'échange entre gravière et nappe? M. PEaudeCERF a-t-il des observations in situ pour confirmer son point de vue ?

Nous n'avons pas d'observations sur ce point, répond M. PEAU DECERF, mais ce problème mérite d'être examiné. On peut penser que, s'il y a un écou'ement provenant de la nappe, le colmatage gravitaire sera peu important; il n'en sera pas forcément de même pour le colmatage biologique ou physico-chimique.

En l'absence d'autres questions, M. le Président clôt la discussion et donne la parole à M. Gallouedec pour l'exposé de sa communication.

\section{Abstract \\ How gravel pits affect hydrodynamic groundwater behaviour}

Alluvial formations are very valuable sources of sand and grave'. They are frequently worked down to groundwater level. The aquifer, which mostly shows promising characteristics, frequently supplies a whole region with drinking water. Quarries in the aquifer system are liable to depress or raise the groundwater level, and especially to affect water qua'ity by chemical or biological factors or by facilitating pollutant inflow. The severity of such effects is directly dependent on the amount of water exchange taking place between the fu'l gravel pit and the aquifer. To evaluate such effects, therefore, it is necessary to establish hydrodynamic relationships existing between the aquifer and surface water.

Author reviews the factors to consider in evaluating such hydrodynamic effects, which include warping of the river banks and bed, depth of excavation and position in relation to the aquifer system, relationships with rivers, shape and dimensions of excavations and position with respect to the general direction of groundwater flow. The effects of these factors vary and can be more clearly visua'ised with the aid of a series of diagrams. River bank and bed warping turns out to be the vital governing factor as regards flow exchange taking place between a gravel pit and aquifers. If warping is taking place the gravel pit is an obstacle to groundwater flow; if not, it acts as a "privileged" flow path.
Present means whereby these parameters can be evaluated are discussed in the second part of the article. Without going into conventional hydrogeo'ogical approaches in detail, special reference is made to local measurement of permeability, vertical permeability distribution through the various ground horizons and, especially, evaluation of river bank and bed warping. Closer methodology, however, seems to be required for some of the points considered.

Interpretation and prediction methods vary from simple caiculation to use of a three-dimensional model. The usefulness of electrical analogies of the conductive paper model type. which are particularly suitable for this type of problem, is also emphasized.

Reference is made to two very different types of investigation. One relates to a gravel pit extension scheme in the Crau region of France, more especially its effects on the local water table level. The other is a more basic study of optimal gravel pit and water supply operation in the Toulouse region, in which the warping problem in quarries and its variation over a period of time are considered. Warping measurements have been carried out on site, from the results of which it is expected that the effects of the gravel pits on present and future water supply yie'ds can be predicted. 